\title{
The Design of ZMP Measure System for Biped Robot
}

\author{
LI Bin ${ }^{1, ~ a, ~ L I ~ Z h i h a i ~}{ }^{2, ~ b}$, LI Jiejia ${ }^{1, ~ c}$, BU Chunguang ${ }^{2, d}$ \\ ${ }^{1}$ School of Mechanical Engineering, Shenyang Jianzhu University, Shenyang 110168, China \\ ${ }^{2}$ State Key Laboratory of Robotics, Shenyang Institute of Automation, Chinese Academy of \\ Sciences, Shenyang 110016, China
}

aleebin1991@hotmail.com, ${ }^{\mathrm{b}}$ lizhihai@sia.cn, ${ }^{\mathrm{c}}$ ljj_01@sjzu.edu.cn, ${ }^{\mathrm{d}} \mathrm{cgbu} @$ sia.cn

\section{Keywords: Zero Moment Point; Stability; Biped Robot}

\begin{abstract}
A kind of ZMP measure system has been described in this paper, tests have been done on person with the system. The obtained experimental ZMP trajectory has been analyzed and real-time performance of this system has also been verified. So, the result has shown that the system can be implemented on our 6 DOF biped robot. The ultimate purpose of this system is to measure ZMP trajectory for further analysis of on-line gait planning of the biped robot.
\end{abstract}

\section{Introduction}

Due to flexibility of bipedal structure, biped robots can go up and down stairs, stride over obstacle and have good performance on complicated task. Biped robots are most suitable robot to be human substitute in the daily environment. However, realization of dynamic stable walking of biped robot is always a challenging task because other than wheeled robot and multi-legged robot, realization of stable walking of biped robot not only requires supreme exquisite mechanical structure, but also appropriate robust control system.

Lots of researchers concentrate on dynamic stable walking of biped robots, so far, there are two methods to make reliable walking. First method is based on algorithm such as neural network and fuzzy control to predetermine ZMP reference, in this way, stable walking can be obtained in given environment. However in the first method, ZMP feedback data is not used and stable walking can be assured only on flat ground, it is also known as off-line gait planning. In second method, the feedback data obtained from attitude sensor, gyroscope, accelerometer, force sensor is used to adjust robot in real-time, so more stable walking can be realized.

Force sensors applied on the sole are necessary, because force distribution and terrain condition which is the important data of biped walking can be obtained through force sensor. Therefore ZMP trajectory can be calculated while robot is walking by feedback data. The purpose of this paper is to obtain the ZMP trajectory which can be used in the further study of stable walking control of biped robot.

\section{Analysis of ZMP}

Biped walk is a periodic process, each leg cross to lift and one foot has to be contact with the ground. For bipedal walking robot, walk can be defined as the bodily movement generated by periodic motion of mechanic joint with the ground reaction forces. There exist two kinds of walking, namely, static walking and dynamic walking. In "static walking", the projection of the center of mass never leaves the support polygon during the walking. In "dynamic walking", there exist periods when the projection of the center of mass leaves the support polygon. So static walking has to be slow to keep the COM projection under polygon and big foot generally used to ensure that. The ZMP theory makes dynamic walking possible, it's faster and more efficient than static walking. We can use flexible control to constrain the attitudes, speed, accelerated speed ensure the balance.

The ZMP is defined as the point where the horizontal component of the moment of the ground reaction forces becomes zero. If ZMP always in the support polygon and close to COP, the walking will be balance, in other term, the walking is dynamic stable. ZMP theory is generally used as criteria for dynamic stable walking of biped robot. So, if ZMP trajectory can be obtained real-time, 
better dynamic stable walking can be realized. The distribution of ground force reaction is also complicated, ground reaction forces distributed over the sole can be replaced by a equivalent force and moment at a certain point in the sole. As shown in Fig 6 and Fig 7.

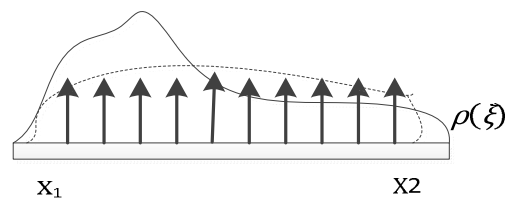

Fig.1 Vertical Force

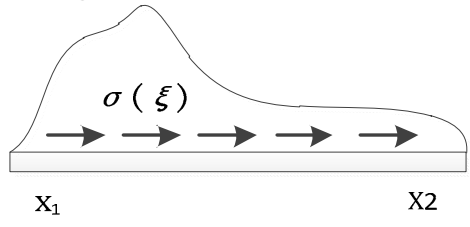

Fig.2 Horizontal Force

The torque $\left(\tau\left(P_{x}\right)\right.$ in the sole can be expressed by:

$\tau\left(P_{x}\right)=\int_{x_{1}}^{x_{2}}\left(\xi-P_{x}\right) \rho(\xi) d \xi$

Considering $\tau\left(P_{x}\right)=0$ for Eq. 1, $P_{x}$ can be obtained as follows:

$P_{x}=\frac{\int_{x_{1}}^{x_{2}} \xi \rho(\xi) d \xi}{\int_{x_{1}}^{x_{2}} \rho(\xi) d \xi}(2)$

So, we can obtain:

$\tau_{p}=\sum_{i=1}^{N}\left(p_{i}-p\right) \times F_{i}$

From Eq. 1- Eq. 3, $P$ can be expressed as follows:

$P=\frac{\sum_{i=1}^{N} P_{i} F_{i}}{\sum_{i=1}^{N} F_{i}}$

Where $F_{i}$ is the pressure force on the sole obtained from the data acquisition system.

As for the force of friction, it can be attributed to resultant force both in single support period and double support period. Here we take no consideration of force of friction. So, we can calculate ZMP using formula as follows:

$$
\begin{aligned}
& P_{x}=\frac{\sum_{i=1}^{N} P_{i x} F_{i z}}{\sum_{i=1}^{N} F_{i z}} \\
& P_{y}=\frac{\sum_{i=1}^{N} P_{i y} F_{i z}}{\sum_{i=1}^{N} F_{i z}}
\end{aligned}
$$

Where $\mathrm{P}_{\mathrm{ix}}$ is the $\mathrm{x}$ coordinate of sensor on the sole, $\mathrm{F}_{\mathrm{iz}}$ is the ground reaction force on the point.

As we know, the process of a periodic walking including two parts, one is single support period, the other is double support period which is short but can't be ignored. So, both SSP and DSP have to be taken into consideration.

The ZMP of double support period as follows:

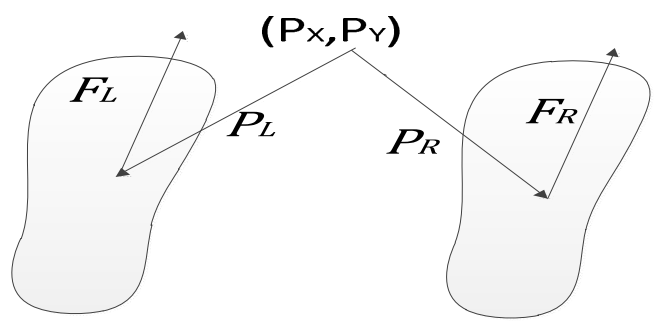

$\mathrm{P}_{\mathrm{X}}=\frac{\mathrm{P}_{\mathrm{Rx}} \mathrm{F}_{\mathrm{Rz}}+\mathrm{P}_{\mathrm{Lx}} \mathrm{F}_{\mathrm{Lz}}}{\mathrm{F}_{\mathrm{Rz}}+\mathrm{F}_{\mathrm{Lz}}}$

Fig .3 ZMP (Double Support Period)

$P_{y}=\frac{P_{R y} F_{R z}+P_{L y} F_{L z}}{F_{R z}+F_{L z}}$

$P_{R x}, P_{R y}$ denotes ZMP of right foot, as the same $P_{L x}, P_{L y}$ denotes the ZMP of left foot. 


\section{Pressure Measurement System Design}

Force sensor is indispensable in feedback system, there are two kinds of sensor mostly used in follow ways: one six-axis force sensor and several normal force sensors. When the ground is uneven, there are many contact points and their positions cannot be measured by a popular 6 axis force sensor, and six-axis force is often applied on the ankle that makes large inertia moment which can produce vibrations. So regular load cells are selected.

In order to realize stable walking of biped robot, the contact force between sole and ground has to be measured precisely, the contact force distribution is most depend on ground condition. In this work four load cells are applied on the corners of biped robot sole. And four spikes of load cells can be assumed as arch of the foot, so the force distribution can be even in flat ground condition. Moreover, pressure can be easily measured.

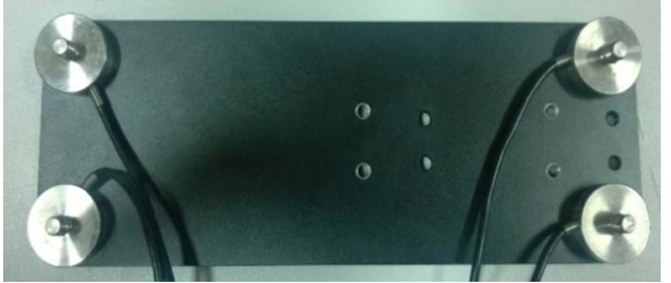

(a)

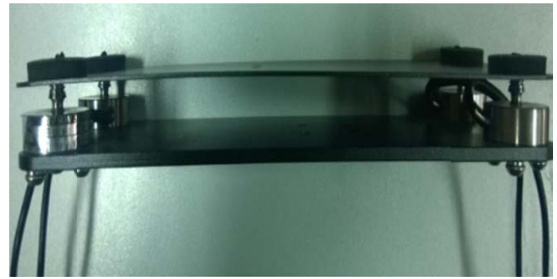

(b)

Fig. 4 ZMP measurement platform

For the convenience of collecting sensor data and fine PCI connection with PC104 board, data acquisition card PCH 2001 has been used, the card has 12 bit resolution, 16 channels, frequency can be as high as $250 \mathrm{KHZ}$ and the output range can cover our load cell output limit. The card can get until 200 samples per second coming from the load cells.

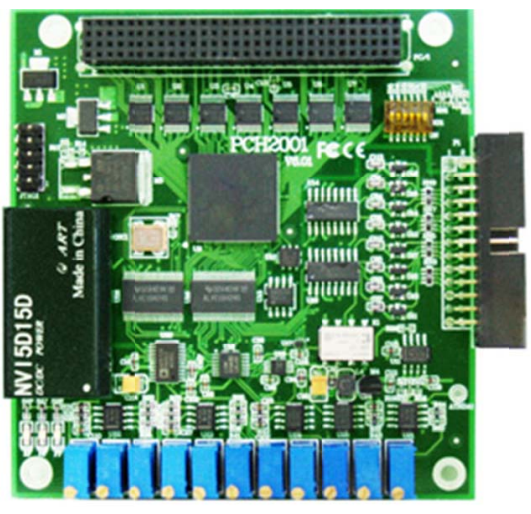

(a)

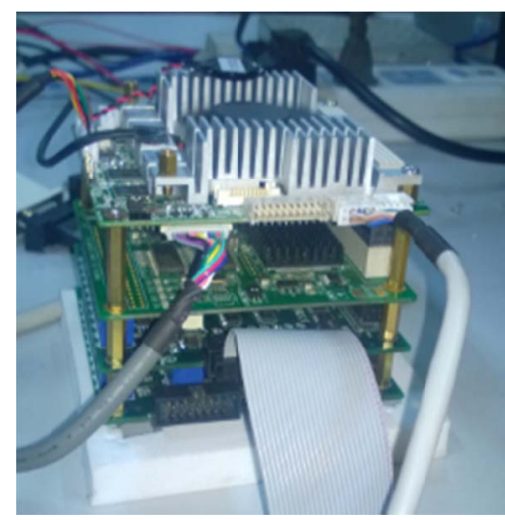

(b)

Fig .5 (a) PCH 2001; (b) Hardware system

Noise filtering has to be done in any signal acquisition system, we have to do the noise filter according to the situation by both hardware and software way. Software filtering is efficient and necessary. According to the feature of this data acquisition system, the filtering algorithm must have good performance on the real-time quality as the whole system does. There are lots of filter methods: Arithmetic average filter is suitable filter for random jamming. Median filter is very efficient to take out fluctuation by accident cause. But random jamming or the instability of transmitters may cause serious distortion we should use program judging method to decide useful signals. So, here we combine three methods above so filtering algorithm is used as follows: 


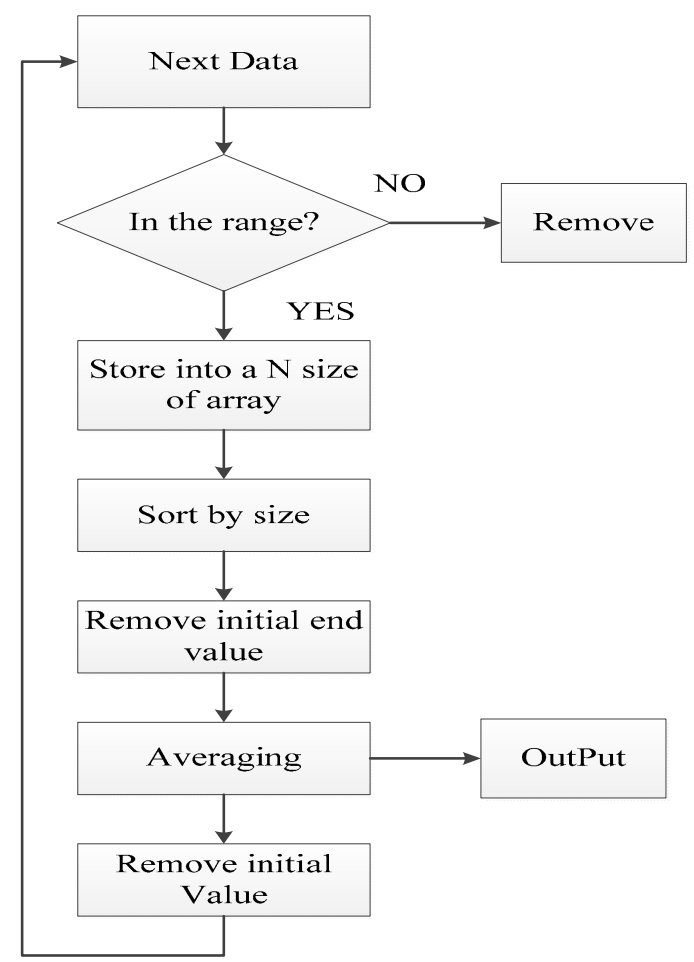

Fig.6 filtering algorithm

Due to the impact force produced by contact between foot and ground, stretchy and damped nonskid rubber has been applied on the contact area on the sole. We can see from Fig.7 that most noisy has been filtered by filtering method used above.

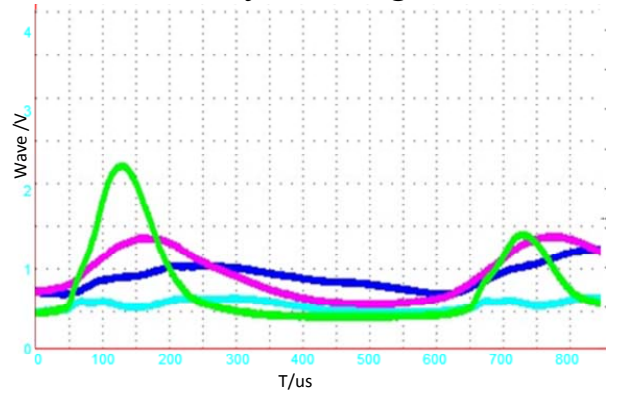

(a)

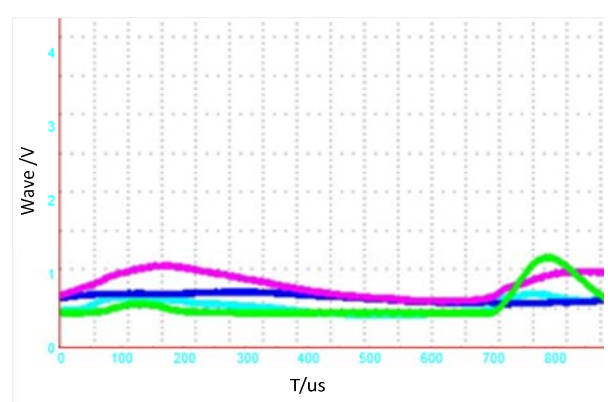

(b)

Fig.7 (a) Pressure value (Before filtering); (b) Pressure value (After filtering)

\section{Experiments and Result}

When a person is starting walking on the platform, the output of load cells will be amplified and converted into digital signals by the acquisition cards. The data will arrive to PC104 board through PCI bus. Finally we filter the signal and calculate the ZMP in the program operated on PC 104 board.

The person walks straight on the floor with $20 \mathrm{~cm}$ step size, The ZMP trajectory of the person is shown in Fig.8.It shows the continuous trajectory of the ZMP for three steps: two steps by the left leg and one step by the right leg. The curve line shows the locus of the ZMP during the period of one-leg support, and the straight line shows that from one one-leg-support period to the subsequent one-leg support period. We can see that the ZMP never leaves support area. 


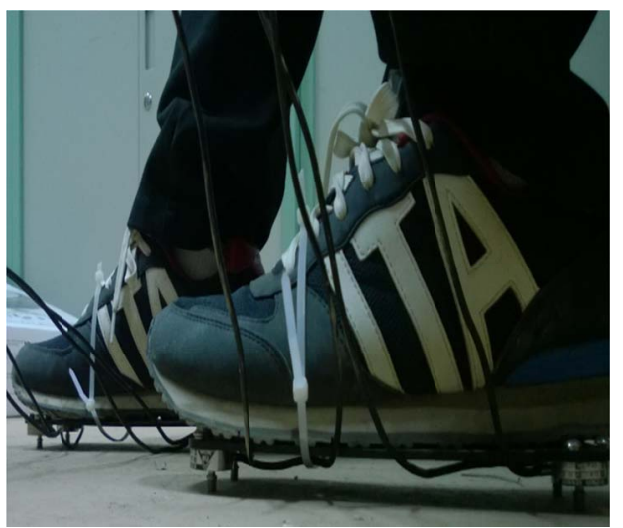

(a)

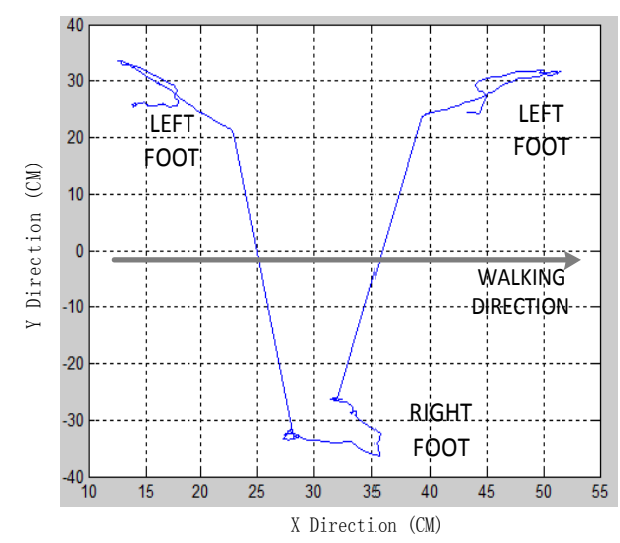

(b)

Fig.8 (a) Experiment of walking; (b) ZMP trajectory

\section{Conclusion}

We use PC 104, data acquisition, load cells to build a measure system, then analyzed the ZMP distribution and use software filter method to reduce noise sensitivity of the system, finally ZMP system has been designed and experiments have been done while person is walking.As we can see from test result that the system offers us accurate, continuous ZMP trajectory and the system has good performance on real-time and accuracy. The system provides conditions for further study of on-line gait planning.

\section{Acknowledgement}

In this paper, the research was sponsored by the National Key Technology Support Program (Project No. 2013BAK03B01).

\section{References}

[1] Shuuji Kajita, Hirohisa Hirukawa, Kensuke Harada: Introduction to Humanoid Robotics.(2006)

[2] JING Chenglin, LI Zushu, XUE Fangzheng: Fuzzy Adaptive Algorithm for Biped Robot Inverse Kinematics, ROBOT 2010,Vol.32(4):534-539,546

[3] Hashlamon, Iyad; Erbatur, Kemalettin: Simple Virtual Slip Force Sensor for Walking Biped Robots,2013 9TH ASIAN CONTROL CONFERENCE (ASCC)

[4] Makoto Shimojo, Takuma Araki: A ZMP sensor for biped robot. 2011 IEEE International Conference on Robotics and Automation

[5] Tatsuzo Ishida, and Yoshihiro Kuroki in: Theoretical Methods in Sensor system of a small biped entertainment robot, volume 5 of Advanced Robotics, Vol. 18, No. 10, pp. 1039-1052 (2004)

[6] M. Arbulú, F. Prieto, L. Cabas, P. Staroverov, D. Kaynov, C. Balaguer: ZMP Human Measure System(2010)

[7] Departamento de Informática, YABIRO: A NEW APROACH TO SMALL BIPED ROBOTS, 5th IFAC/EURON Symposium on Intelligent Autonomous Vehicles Instituto Superior Técnico, Lisboa, Portugal July 5-7, 2004 